\title{
Theorems on gravitational time delay and related issues
}

\author{
Sijie Gao and Robert M. Wald \\ Enrico Fermi Institute and Department of Physics \\ University of Chicago \\ 5640 S. Ellis Avenue \\ Chicago, Illinois 60637-1433
}

October 23, 2018

\begin{abstract}
Two theorems related to gravitational time delay are proven. Both theorems apply to spacetimes satisfying the null energy condition and the null generic condition. The first theorem states that if the spacetime is null geodesically complete, then given any compact set $K$, there exists another compact set $K^{\prime}$ such that for any $p, q \notin K^{\prime}$, if there exists a "fastest null geodesic", $\gamma$, between $p$ and $q$, then $\gamma$ cannot enter $K$. As an application of this theorem, we show that if, in addition, the spacetime is globally hyperbolic with a compact Cauchy surface, then any observer at sufficiently late times cannot have a particle horizon. The second theorem states that if a timelike conformal boundary can be attached to the spacetime such that the spacetime with boundary satisfies strong causality as well as a compactness condition, then any "fastest null geodesic" connecting two points on the boundary must lie entirely within the boundary. It follows from this theorem that generic perturbations of anti-de Sitter spacetime always produce a time delay relative to anti-de Sitter spacetime itself.
\end{abstract}




\section{Introduction}

Gravitational time delay effects in general relativity have been considered by many authors. Although general relativity makes unambiguous predictions for, e.g., the time of reception of signals sent by a space probe orbiting nearly behind the sun - and these predictions have been observationally verified [1] - it appears remarkably difficult to characterize these effects in a meaningful way in terms of the "time delay" of a light ray relative to the time required in Minkowski spacetime. The reason for this difficulty is that, in general, there is no natural way to choose a flat background for a curved spacetime, and, thus, no meaningful way to compare the propagation of a light ray in a curved spacetime with that of a "corresponding" light ray in Minkowski spacetime [2].

Recently, a general result on gravitational time delay was obtained in [3]. The authors of [3] considered linearized perturbations

$$
h_{a b}=g_{a b}-\eta_{a b}
$$

of Minkowski spacetime that satisfy the linearized version of the the null energy condition. (The null energy condition states that

$$
R_{a b} k^{a} k^{b} \geq 0
$$

for all null $k^{a}$.) It was further required that no incoming gravitational radiation be present. It was then shown that in the Lorentz gauge,

$$
\partial_{b}\left[h^{a b}-\frac{1}{2} \eta^{a b} h\right]=0
$$

the metric perturbation satisfies

$$
h_{a b} k^{a} k^{b} \geq 0
$$

for all $k^{a}$ that are null with respect to the Minkowski background, i.e.,

$\eta_{a b} k^{a} k^{b}=0$. Eq.(4) can be interpreted as saying that the light cones of the perturbed metric contract relative to the flat background, i.e., the propagation of light is "slower" in the perturbed spacetime.

However, the above result is highly gauge dependent, i.e., it depends in a crucial way on how one chooses to compare the perturbed spacetime with 
the background Minkowski spacetime. The choice of a different manner of comparison corresponds to changing $h_{a b}$ via a gauge transformation

$$
h_{a b} \rightarrow h_{a b}+\partial_{a} \xi_{b}+\partial_{b} \xi_{a}
$$

The following is an explicit example of a smooth, pure gauge $h_{a b}$ that "opens out" the light cones everywhere and also goes to zero as $1 / r$ at spatial and null infinity: In spherical polar coordinates in Minkowski spacetime, let

$$
\xi_{a}=-\frac{r_{a}}{2+g(r)}
$$

where $r^{a}$ is the radially outward pointing vector with $r^{a} r_{a}=r^{2}$ (i.e., $r_{a}=$ $\left.r(d r)_{a}\right)$ and $g$ is any smooth function such that $g(r)=r$ for $r \geq 1, g(r)=r^{2}$ for $0 \leq r \leq 1 / 2$, and $g(r) \geq 0,0 \leq g^{\prime}(r)<2$ for all $r$. Then, we have

$$
\begin{aligned}
h_{a b} & =2 \partial_{(a} \xi_{b)} \\
& =\frac{2 g^{\prime}(r)}{[2+g(r)]^{2}}(d r)_{a} r_{b}-\frac{2}{2+g(r)} \partial_{(a} r_{b)} \\
& =\frac{2 r g^{\prime}(r)}{[2+g(r)]^{2}}(d r)_{a}(d r)_{b}-\frac{2}{2+g(r)} q_{a b}
\end{aligned}
$$

where $q_{a b}$ is the Euclidean metric on the $t=$ constant hypersurfaces. Since $g(r)=r$ for $r \geq 1$, we see that the orthonormal frame components of $h_{a b}$ are $O(1 / r)$ as $r \rightarrow \infty$ (independently of $t$ ). For any null vector $k^{a}$, we have

$$
\begin{aligned}
h_{a b} k^{a} k^{b} & =\frac{2 r g^{\prime}(r)}{[2+g(r)]^{2}}\left(\frac{\vec{k} \cdot \vec{x}}{r}\right)^{2}-\frac{2}{2+g(r)}|\vec{k}|^{2} \\
& <0
\end{aligned}
$$

i.e., this pure gauge $h_{a b}$ opens out the light cones everywhere. By adding a suitable multiple of this pure gauge perturbation to the perturbation considered in [3], we can reverse the conclusions of [3] in any compact region of spacetime.

Indeed, a theorem of Penrose [5] proves that it is impossible to identify Schwarzschild spacetime with Minkowski spacetime so that the null infinities of the two spacetimes coincide and the light cones of the Schwarzschild metric lie within the lightcones of the Minkowski metric. (This does not contradict the results of [3] because the Lorentz gauge is ill behaved at null infinity [6].) 
From the point of view taken in [3], Penrose's result might be interpreted as an "anti-time-delay" theorem, but it would seem more reasonable to interpret both Penrose's theorem and the results of [3] as having more to do with the manner in which the identification of the curved and flat spacetimes are made than with gravitational time delay.

As pointed out by Olum [四], in the case of a spacetime that is Minkowskian outside of a world tube (see Fig. 2 of [4]), an unambiguous comparison with Minkowski spacetime can be done in the Minkowskian region, so the notion of "time delay" is well defined in this context. It was shown in 1 that in such a spacetime, if the null energy condition (2) and null generic condition (see eq.(9) below) hold within the non-flat region, then a "time advance" cannot occur. However, if the dominant energy condition holds, it is impossible to have a spacetime (other than Minkowski spacetime) that is Minkowskian outside of a worldtube, since the ADM mass of such a spacetime would vanish, in contradiction with the positive mass theorem. In [4], an alternative characterization of "time delay" also was proposed. This characterization does not require a comparison with Minkowski spacetime, and a time delay theorem was proven for this definition.

In this paper, we will prove two theorems related to "time delay" that also do not require a comparison with Minkowski spacetime. Both theorems apply to spacetimes that satisfy the null energy condition (2) as well as the null generic condition. (The null generic condition is the statement that each null geodesic contains a point at which

$$
k_{[a} R_{b] c d[e} k_{f]} k^{c} k^{d} \neq 0
$$

where $k^{a}$ denotes the tangent to the geodesic.) It appears likely that our assumption that the null generic condition holds could be significantly weakened by use of the null splitting theorems of [7], but we shall not consider this issue further here.

Our first theorem states that in a null geodesically complete spacetime satisfying the null energy condition and the null generic condition, given any compact set $K$, there exists another compact set $K^{\prime}$ satisfying the following property: If $p$ and $q$ are any two events lying outside of $K^{\prime}$ such that $q \in J^{+}(p)-I^{+}(p)$, then any causal curve, $\gamma$, connecting $p$ with $q$ (which necessarily must be a null geodesic) cannot enter $K$ (see Fig. 1). The relationship of this result to "time delay" is as follows: If it were possible to arrange the spacetime geometry in a compact region $K$ so as to produce a 


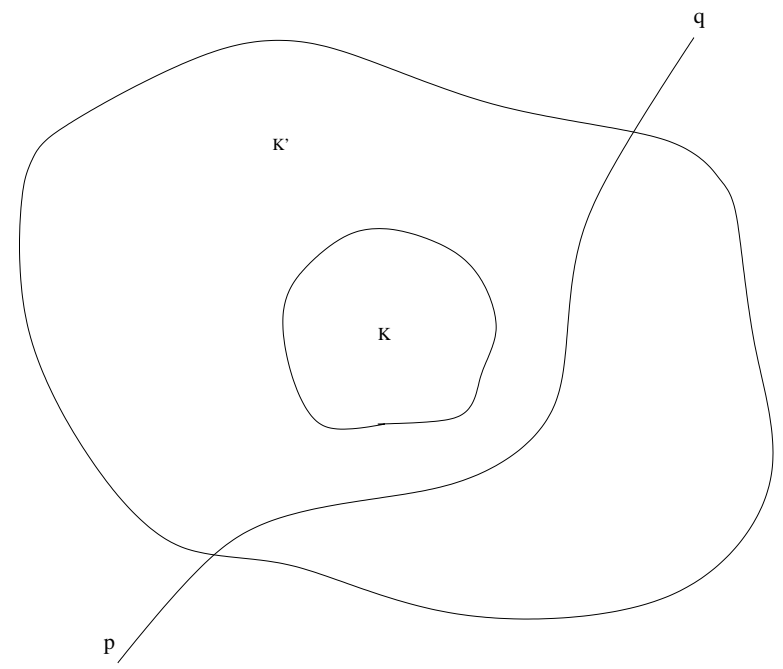

Figure 1: In a null geodesically complete spacetime satisfying the null energy condition and the null generic condition, given a compact region $K$, there exists another compact region $K^{\prime}$ such that if $p, q \notin K^{\prime}$ and $q \in J^{+}(p)-I^{+}(p)$, then any causal curve connecting $p$ to $q$ cannot intersect $K$.

"time advance", then one might expect a "fastest null geodesic" $\gamma$ to take advantage of this by entering $K$. Thus, the theorem suggests that "time advance" is not possible, although it is difficult to make a strong argument for this interpretation, since the theorem gives little control over the size of the region $K^{\prime}$. Some additional applications of this theorem will be given in section 2 .

In section 3, we present our second theorem. In this theorem, we restrict attention to spacetimes satisfying the null energy condition and null generic condition to which a timelike conformal boundary can be attached, such that the conformally completed spacetime, $\bar{M}$, satisfies strong causality together with the property that for all $p, q \in \bar{M}$, the set $J^{+}(p) \cap J^{-}(q) \cap \bar{M}$ is compact. The prototype spacetimes satisfying these properties are asymptotically antide Sitter spacetimes, although anti-de Sitter spacetime itself would not be in this class on account of its failure to satisfy the null generic condition. For the spacetimes satisfying the hypotheses of this theorem, we prove that any "fastest null geodesic" in $\bar{M}$ connecting two points $p, q$ in the conformal boundary, $\dot{M}$, must lie entirely within $\dot{M}$. Now, in the case of anti-de Sitter spacetime itself, it turns out that if the points $p, q \in \dot{M}$ are the past and 
future endpoints in $\bar{M}$ of a null geodesic in $M$, then there also exists a null geodesic in $\dot{M}$ connecting $p$ and $q$, but no "faster" null geodesic exists, i.e., $q$ lies on the boundary of the future of $p$ in $\bar{M}$. In other words, in anti-de Sitter spacetime, in a "race" to get from $p \in \dot{M}$ to a point in $\dot{M}$ antipodal to $p$, there is a "tie" between null geodesics passing through $M$ and null geodesics lying in $\dot{M}$. Our theorem proves that in a generically perturbed anti-de Sitter spacetime, this "tie" is always broken in favor of geodesics lying within the boundary. Thus, our theorem can be interpreted as saying that in generically perturbed anti-de Sitter spacetime, there is always a "time delay" relative to anti-de Sitter spacetime itself. A similar result under somewhat different hypotheses has previously been obtained by Woolgar [8].

Our notation and conventions throughout this paper follow those of [2]. All spacetimes considered in this paper will be assumed to be connected, smooth, time oriented, and paracompact. No assumption will be made about the dimensionality of spacetime, i.e., our results hold in any spacetime dimension.

\section{Avoidance of Compact Sets by Sufficiently Long "Fastest Null Geodesics"}

In this section, we will prove the following theorem and then discuss some applications of it.

Theorem 1 Let $\left(M, g_{a b}\right)$ be a null geodesically complete spacetime satisfying

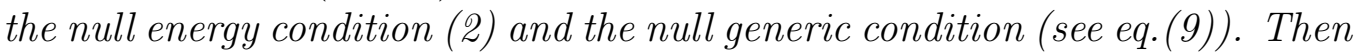
given any compact region $K \subset M$, there exists another compact region $K^{\prime}$ containing $K$ such that if $q, p \notin K^{\prime}$ and $q \in J^{+}(p)-I^{+}(p)$, then any causal curve $\gamma$ connecting $p$ to $q$ cannot intersect the region $K$.

\footnotetext{
${ }^{1}$ Recall that a null line is an inextendible, achronal null geodesic. G. Galloway (private communication) has pointed out to us that the conclusions of Theorem 1 remain valid if the hypothesis of Theorem 1 is replaced by the condition that $\left(M, g_{a b}\right)$ fails to contain a null line. Since the present hypotheses of Theorem 1 (i.e., null geodesic completeness, the null energy condition, and the null generic condition) preclude the existence of a null line, Galloway's modification of Theorem 1 is a stronger result than our version. Galloway's proof of his version of Theorem 1 provides a direct construction of a null line when the conclusion of Theorem 1 fails, thereby bypassing Lemma 1 (whose proof makes explicit use of the null energy condition).
} 
The proof of this theorem, will be based upon a lemma that we shall state and prove below. Before doing so we introduce some notation and recall some facts about conjugate points. Since $M$ is time oriented, we may choose a continuous, nowhere vanishing, future-directed timelike vector field $t^{a}$. We define

$$
\mathcal{S}=\left\{\left(p, k^{a}\right) \mid p \in M, k^{a} \in V_{p}, k^{a} k_{a}=0, k^{a} t_{a}=-1\right\}
$$

where $V_{p}$ denotes the tangent space at $p$. Thus, $\mathcal{S}$ consists of the points in the tangent bundle of $M$ where the tangent vector is a future directed null vector that is normalized with respect to $t^{a}$. We shall denote points of $\mathcal{S}$ by $\Lambda$. Associated with any $\Lambda=\left(p, k^{a}\right) \in \mathcal{S}$ is the null geodesic $\gamma_{\Lambda}$ which starts at $p$ with tangent $k^{a}$; we take the affine parameter, $\lambda$, of $p$ along $\gamma_{\Lambda}$ to be 0 , i.e., $\gamma_{\Lambda}(0)=p$.

Recall that points $p, q$ along a geodesic $\gamma$ are said to be conjugate if there exists a Jacobi field which vanishes at both $p$ and $q$. Equivalently (see, e.g., [9] or [2]), if we define the matrix $A^{\mu}{ }_{\nu}(\lambda)$ at affine parameter $\lambda$ along $\gamma$ by

$$
\frac{d^{2} A^{\mu}{ }_{\nu}}{d^{2} \lambda}=-\sum_{\alpha, \beta, \sigma} R_{\alpha \beta \sigma}{ }^{\mu} k^{\alpha} k^{\sigma} A^{\beta}{ }_{\nu}
$$

with initial conditions $\left.A^{\mu}{ }_{\nu}\right|_{p}=0$ and $\left.\left(d A^{\mu}{ }_{\nu} / d \lambda\right)\right|_{p}=\delta^{\mu}{ }_{\nu}$, then $q$ will be conjugate to $p$ if and only if $\operatorname{det} A=0$ at $q$. We define

$$
G(\lambda)=\sqrt{\operatorname{det} A(\lambda)}
$$

Since $A^{\mu}{ }_{\nu}$ is a solution to the linear ordinary differential equation (11), it follows that $A^{\mu}{ }_{\nu}$ and hence $\operatorname{det} A$ vary smoothly with $(\Lambda, \lambda)$. Consequently, $G$ varies smoothly with $(\Lambda, \lambda)$ except possibly at points where $G=0$. Finally, we recall that in terms of $G$, the Raychaudhuri equation yields (see, e.g., [10])

$$
G^{\prime \prime} / G=-\frac{1}{2}\left[\sigma_{a b} \sigma^{a b}+R_{a b} k^{a} k^{b}\right]
$$

where $\sigma_{a b}$ denotes the shear of the congruence of null geodesics emanating from $p$.

Lemma 1 Let $\left(M, g_{a b}\right)$ satisfy the null energy condition (but not necessarily be null geodesically complete nor satisfy the null generic condition). Let $\Lambda_{0}=\left(p_{0}, k_{0}^{a}\right) \in \mathcal{S}$ be such that the null geodesic $\gamma_{\Lambda_{0}}$ possesses a conjugate 
point to $p_{0}$, which, for definiteness, we assume occurs at a positive value of $\lambda$, i.e., to the future of $p_{0}$. Then there exists an open neighborhood $O \subset \mathcal{S}$ of $\Lambda_{0}$ such that for all $\Lambda=\left(p, k^{a}\right) \in O$, the null geodesic $\gamma_{\Lambda}$ will possess a conjugate point to the future of $p$. Furthermore, if we define the map $h: O \rightarrow M$ by $\Lambda=\left(p, k^{a}\right) \mapsto h(p)$, where $h(p)$ is the first conjugate point to the future of $p$ along $\gamma_{\Lambda}$, then $h$ is continuous at $\Lambda_{0}$.

Proof of Lemma 1: Let $q_{0}$ denote the first conjugate point to $p_{0}$ along $\gamma_{\Lambda_{0}}$ in the future direction, i.e., the conjugate point to $p_{0}$ lying at the smallest positive value of affine parameter. Let $\lambda_{0}>0$ denote the affine parameter value of $q_{0}$. Since the exponential map is defined on an open subset of the tangent bundle, it follows that there is an open neighborhood $\tilde{O} \subset \mathcal{S}$ of $\Lambda_{0}$ and an $\epsilon>0$ such that all null geodesics determined by initial data in $\tilde{O}$ extend at least to affine parameter $\lambda_{0}+\epsilon$. We wish to show that given any open neighborhood $U \subset M$ of $q_{0}$, there exists an open neighborhood $O \subset \tilde{O}$ of $\Lambda_{0}$ such that for all $\Lambda \in O$, the function $G$ on $\gamma_{\Lambda}$ defined by eq.(12) above will vanish for the first time at a point lying in $U$. By doing so, we will establish the existence of a conjugate point to $p$ on $\gamma_{\Lambda}$ and prove the continuity of the map $h$.

Define the map $H: \tilde{O} \times\left[0, \lambda_{0}+\epsilon\right] \rightarrow M$ by $(\Lambda, \lambda) \mapsto \gamma_{\Lambda}(\lambda)$. Then $H$ is continuous. Therefore, given an open neighborhood $U \subset M$ of $q_{0}$, there exists an open neighborhood $O^{\prime} \subset \tilde{O}$ of $\Lambda_{0}$ and a $\delta>0$ (with $\delta<\epsilon$ ) such that $H(\Lambda, \lambda) \in U$ whenever $\Lambda \in O^{\prime}$ and $\left|\lambda-\lambda_{0}\right|<\delta$. Therefore, the lemma will be proven if we can find an open neighborhood $O \subset O^{\prime}$ of $\Lambda_{0}$, such that for all $\Lambda \in O$, the first positive value of $\lambda$ at which the function $G(\Lambda, \lambda)$ vanishes lies within $\delta$ of $\lambda_{0}$.

By hypothesis, $G\left(\Lambda_{0}, \lambda_{0}\right)=0$ and $G\left(\Lambda_{0}, \lambda\right)>0$ for all $0<\lambda<\lambda_{0}$. It follows immediately from eq. (13) and the null energy condition that $G^{\prime \prime}\left(\Lambda_{0}, \lambda\right) \leq$ 0 for all $0<\lambda<\lambda_{0}$. By the mean value theorem applied to $G$, there must exist $\lambda_{1} \in\left(0, \lambda_{0}\right)$ such that $G^{\prime}\left(\Lambda_{0}, \lambda_{1}\right)=-C$ for some $C>0$. Since $G^{\prime \prime} \leq 0$, it follows that $G^{\prime}\left(\Lambda_{0}, \lambda\right) \leq-C$ for all $\lambda \in\left[\lambda_{1}, \lambda_{0}\right)$. By choosing $\lambda_{1}$ to be sufficiently near $\lambda_{0}$, we may assume without loss of generality that $\lambda_{0}-\lambda_{1}<\delta$ and

$$
\frac{G\left(\Lambda_{0}, \lambda_{1}\right)}{\left|G^{\prime}\left(\Lambda_{0}, \lambda_{1}\right)\right|}<\delta
$$

since $G\left(\Lambda_{0}, \lambda_{1}\right) \rightarrow 0$ as $\lambda_{1} \rightarrow \lambda_{0}$ but $\left|G^{\prime}\left(\Lambda_{0}, \lambda_{1}\right)\right|$ remains bounded below by $C$. 
From the continuous dependence of $G$ and its derivatives on $\Lambda$, it follows that there exists an open neighborhood $O \subset O^{\prime}$ of $\Lambda_{0}$, such that for all $\Lambda \in O$, we have (i) $G(\Lambda, \lambda)>0$ for all $0<\lambda<\lambda_{1}$, (ii) $G^{\prime}\left(\Lambda, \lambda_{1}\right)<0$, and (iii) $G\left(\Lambda, \lambda_{1}\right) /\left|G^{\prime}\left(\Lambda, \lambda_{1}\right)\right|<\delta$. Since $G^{\prime \prime}<0$ by eq.(13), it follows that for all $\Lambda \in O, G(\Lambda, \lambda)$ must achieve its first zero between $\lambda_{1}$ and $\lambda_{1}+\delta$. Since $\lambda_{0}-\delta<\lambda_{1}<\lambda_{0}$, this implies that $G(\Lambda, \lambda)$ must achieve its first zero within $\delta$ of $\lambda_{0}$, as we desired to show.

Proof of Theorem 1: Since $M$ is assumed to be paracompact, we may introduce a Riemannian metric $q_{a b}$ on $M$ (see, e.g., Appendix 2 of [11]). By multiplying $q_{a b}$ by a conformal factor if necessary, we may assume without loss of generality that $q_{a b}$ is complete (see Thm. 17 of [12]). Choose a point $x \in M$ and let $r: M \rightarrow \mathbb{R}$ denote the geodesic distance from $x$ in the metric $q_{a b}$. Then $r$ is a continuous function on $M$ and for all $R>0$, the set $B_{R}=\{p \in M \mid r(p) \leq R\}$ is compact (see Thm. 15 of [12]).

Since $M$ is null geodesically complete and satisfies the null energy condition and null generic condition, it follows that every null geodesic in $M$ contains a pair of conjugate points [9]. As discussed above, associated with each $\Lambda \in \mathcal{S}$ is a null geodesic $\gamma_{\Lambda}$ determined by the initial conditions $\Lambda$. Define $f: \mathcal{S} \rightarrow \mathbb{R}$ by

$$
\begin{aligned}
f(\Lambda)= & \left\{\inf R \mid B_{R} \text { contains a connected segment of } \gamma_{\Lambda}\right. \text { that } \\
& \text { includes the initial point determined by } \Lambda \text { together } \\
& \text { with a pair of conjugate points of } \left.\gamma_{\Lambda}\right\}
\end{aligned}
$$

We claim that $f$ is an upper-semicontinuous function. To prove this, we must show that given any $\Lambda_{1} \in \mathcal{S}$ and given any $\epsilon>0$, there exists an open neighborhood, $O_{1}$, of $\Lambda_{1}$ such that for all $\Lambda \in O_{1}$ we have $f(\Lambda) \leq f\left(\Lambda_{1}\right)+\epsilon$. Let $p, q$ be conjugate points along $\gamma_{\Lambda_{1}}$ such that a connected segment of $\gamma_{\Lambda}$ containing $p, q$, together with the initial point lies in $B_{R}$ with $R=f\left(\Lambda_{1}\right)+\epsilon / 3$. Without loss of generality, we may assume that $q$ is the first conjugate point to $p$ encountered along $\gamma_{\Lambda_{1}}$ starting at $p$. Let $\Lambda_{0}=\left(p, k^{a}\right)$ denote the initial data for $\gamma_{\Lambda_{1}}$ viewed as a geodesic starting at $p$ (with its tangent re-scaled, if necessary, so as to meet the normalization condition of (10)). With $\gamma_{\Lambda_{1}}$ viewed in this manner, let $\lambda_{0}$ denote the affine parameter of $q$. By the proof of Lemma 1 above, given any $\delta>0$, we can find an open neighborhood, $O_{0} \subset \mathcal{S}$, of $\Lambda_{0}$ such that the null geodesic starting at any $\left(s, l^{a}\right) \in O_{0}$, will have a conjugate point to $s$ within affine parameter $\lambda_{0}+\delta$ of $s$. Choose $\delta$ to 
be sufficiently small that the segment of $\gamma_{\Lambda_{1}}$ between $\lambda_{0}$ and $\lambda_{0}+\delta$ lies within the ball of radius $f\left(\Lambda_{1}\right)+2 \epsilon / 3$. By the continuity of the exponential map, without loss of generality we may then assume that $O_{0}$ is sufficiently small that for all $\left(s, l^{a}\right) \in O_{0}$, the entire null geodesic segment starting at $s$ and ending at affine parameter $\lambda_{0}+\delta$ will lie in a ball of radius $f\left(\Lambda_{1}\right)+\epsilon$. Finally, appealing again to the continuity of the exponential map, we may choose $O_{1}$ to be such that (i) for all $\Lambda \in O_{1}$, the segment of $\gamma_{\Lambda}$ between affine parameter 0 and $\lambda_{1}$ lies in the ball of radius $f\left(\Lambda_{1}\right)+\epsilon$, where $\lambda_{1}$ denotes the affine parameter at which $\gamma_{\Lambda_{1}}\left(\lambda_{1}\right)=p$, and (ii) the initial data for the geodesic $\gamma_{\Lambda}$ at affine parameter $\lambda_{1}$ corresponds to a point in $O_{0}$. It then follows that for all $\Lambda \in O_{1}$, a connected segment of $\gamma_{\Lambda}$ containing the initial point together with a pair of conjugate points will be contained within the ball of radius $f\left(\Lambda_{1}\right)+\epsilon$. Consequently $f(\Lambda) \leq f\left(\Lambda_{1}\right)+\epsilon$.

Let $K \subset M$ be compact. Let $\mathcal{S}_{K}=\left\{\left(p, k^{a}\right) \in \mathcal{S} \mid p \in K\right\}$. Then $\mathcal{S}_{K}$ is compact. Therefore, since $f$ is upper-semicontinuous, it must achieve a maximum, $\alpha$, on $\mathcal{S}_{K}$. Let $K^{\prime}=B_{\alpha}$. Suppose that $q, p \notin K^{\prime}$ and $q \in$ $J^{+}(p)-I^{+}(p)$. Let $\gamma$ be a causal curve with past endpoint $p$ and future endpoint $q$. Then $\gamma$ must be a null geodesic that does not contain any pair of conjugate points lying between $p$ and $q$ (see, e.g., [9], [2]). However, if $\gamma$ intersects $K$, then by the above argument, it necessarily contains a pair of conjugate points lying in $K^{\prime}$ and, hence, lying between $p$ and $q$. Consequently, $\gamma$ cannot intersect $K \square$.

As already indicated in the Introduction, Theorem 1 contains some suggestion of a general "time delay" phenomena in general relativity, since if it were possible to produce a "time advance" in a compact region $K$, then one might expect a "fastest null geodesic", $\gamma$, to take advantage of this by entering $K$. However, since $K^{\prime}$ could be far larger than $K$, it is difficult to make a strong argument for this kind of interpretation of the theorem.

It should be noted that in the case of asymptotically flat spacetimes, Theorem 1 expresses a key aspect of the argument found in the Penrose-SorkinWoolgar [13 positive mass theorem. In an asymptotically flat spacetime, consider a sequence of points $p_{n}, q_{n}$ with $q_{n} \in J^{+}\left(p_{n}\right)-I^{+}\left(p_{n}\right)$ such that both $\left\{p_{n}\right\}$ and $\left\{q_{n}\right\}$ approach infinity as $n \rightarrow \infty$. Then Theorem 1 implies that given any compact set $K$ of the spacetime, for sufficiently large $n$ the corresponding sequence of causal curves $\left\{\gamma_{n}\right\}$ cannot enter $K$, i.e., for sufficiently large $n$, the entire curve $\gamma_{n}$ must lie arbitrarily near infinity. The Penrose-Sorkin-Woolgar theorem is obtained by showing that this behavior 
is incompatible with a negative value of the mass of the spacetime.

Theorem 1 also contains some implications not directly related to "time delay". The following Corollary|2 establishes the absence of "particle horizons" in a class of cosmological models.

Corollary 1 Let $\left(M, g_{a b}\right)$ have the properties stated in the theorem, i.e., suppose that $\left(M, g_{a b}\right)$ is null geodesically complete and satisfies the null energy condition and the null generic condition. Suppose, in addition, that $\left(M, g_{a b}\right)$ is globally hyperbolic, with a compact Cauchy surface $\Sigma$. Then there exist Cauchy surfaces $\Sigma_{1}$ and $\Sigma_{2}$ (with $\Sigma_{2} \subset I^{+}\left(\Sigma_{1}\right)$ ) such that if $q \in I^{+}\left(\Sigma_{2}\right)$, then $\Sigma_{1} \subset I^{-}(q)$.

Proof: Since $\left(M, g_{a b}\right)$ is globally hyperbolic, there exists a continuous "global time function", $T: M \rightarrow \mathbb{R}$, such that each surface of constant $T$ is a Cauchy surface (see, e.g., [2]). Let $K=\Sigma$, and let $K^{\prime}$ be as in Theorem 1. Let $T_{1}$ and $T_{2}$ denote, respectively, the minmum and maximum values of $T$ on $K^{\prime}$. Let $\Sigma_{1}$ be any Cauchy surface with $T<T_{1}$ and let $\Sigma_{2}$ denote the Cauchy surface $T=T_{2}$. Let $q \in I^{+}\left(\Sigma_{2}\right), p \in \Sigma_{1}$ and suppose that $p \in \dot{I}^{-}(q)$. Since $\left(M, g_{a b}\right)$ is globally hyperbolic, $J^{-}(q)$ is closed (see, e.g., [2]), so $p \in J^{-}(q)-I^{-}(q)$, and thus there is a causal curve connecting $p$ to $q$. It follows from Theorem 1 that this causal curve does not intersect $\Sigma$. However, this contradicts the fact that $\Sigma$ is a Cauchy surface. Consequently, there cannot exist a $p \in \Sigma_{1}$ and such that $p \in \dot{I}^{-}(q)$, i.e., $\dot{I}^{-}(q) \cap \Sigma_{1}=\emptyset$. However, $I^{-}(q)$ is open, and since $\dot{I}^{-}(q) \cap \Sigma_{1}=\emptyset$, the complement of $I^{-}(q)$ in $\Sigma_{1}$ also is open. Since we have $I^{-}(q) \cap \Sigma_{1} \neq \emptyset$, and $\Sigma_{1}$ is connected (since $M$ is assumed to be connected), this implies that $\Sigma_{1} \subset I^{-}(q)$, as we desired to show.

It should be noted that de Sitter spacetime satisfies all the hypotheses of our Corollary except the null generic condition. It is not difficult to verify that for any event $q$ in de Sitter spacetime, $I^{-}(q)$ does not contain any Cauchy surface, i.e., de Sitter spacetime also fails to satisfy the conclusion of our Corollary - although it "just barely" fails in the sense that the past of an observer arbitrarily far in the future will come arbitrarily close to containing a Cauchy surface. However, our Corollary shows that if we slightly perturb

\footnotetext{
${ }^{2}$ In accordance with footnote 1, the conclusions of Corollary 1 continue to hold if the hypotheses that $\left(M, g_{a b}\right)$ is null geodesically complete and satisfies the null energy condition and the null generic condition is replaced by the hypothesis that $\left(M, g_{a b}\right)$ fails to contain a null line.
} 
de Sitter spacetime so that the null generic condition is satisfied, then at sufficiently late times, any observer will be able to "view the entire universe".

\section{Time Delay in Spacetimes with a Timelike Conformal Boundary}

In this section, we shall prove a general theorem that has a direct interpretation as showing that there is a "time delay" in asymptotically anti-de Sitter spacetimes relative to anti-de Sitter spacetime itself. A similar result in the particular context of asymptotically anti-de Sitter spacetimes has been previously obtained by Woolgar [8], who extended the arguments of [13] to the asymptotically anti-de Sitter case.

The general context of our theorem is one in which the physical spacetime of interest, $\left(M, g_{a b}\right)$, can be conformally embedded in a spacetime $\left(\widetilde{M}, \tilde{g}_{a b}\right)$, in such a way that the boundary, $\dot{M}$, of $M$ in $\widetilde{M}$ is a timelike hypersurface. We write $\bar{M}=M \cup \dot{M}$. Unless otherwise stated, in this section all futures and pasts are understood as being taken with respect to $\bar{M}$, so, for example,

$$
\begin{aligned}
J^{+}(p) \equiv & \{q \in \bar{M} \mid \text { there exists a causal curve in } \bar{M} \\
& \text { connecting } p \text { to } q\}
\end{aligned}
$$

For $p \in \dot{M}$, we also shall be interested in considering the events in $\dot{M}$ that can be reached by causal curves starting at $p$ that lie entirely in $M$ except for their endpoints. For $p \in \dot{M}$, we define

$$
\begin{aligned}
A(p)= & \{r \in \dot{M} \mid \text { there exists a future directed causal curve } \lambda \text { starting } \\
& \text { from } p \text { and ending at } r \text { satisfying } \lambda-p \cup r \subset M\}
\end{aligned}
$$

We denote the boundary of $A(p)$ in $\dot{M}$ by $\dot{A}(p)$.

Theorem 2 Suppose $\left(M, g_{a b}\right)$ can be conformally embedded in a spacetime $\left(\widetilde{M}, \tilde{g}_{a b}\right)$, so that in $M$ we have $\tilde{g}_{a b}=\Omega^{2} g_{a b}$ and on $\dot{M}$ we have $\Omega=0$, where $\Omega$ is smooth on $\widetilde{M}$. Suppose $\left(M, g_{a b}\right)$ satisfies the following conditions: (1) $\left(M, g_{a b}\right)$ satisfies the null energy condition and the null generic condition. (2) $\bar{M}$ is strongly causal. (3) For any $p, q \in \bar{M}, J^{+}(p) \cap J^{-}(q)$ is compact.

\footnotetext{
${ }^{3}$ The null energy condition could be replaced here by the weaker, averaged condition appearing in a theorem of Borde [14].
} 
(4) $\dot{M}$ is a timelike hypersurface in $\widetilde{M}$. Let $p \in \dot{M}$. Then, for any $q \in \dot{A}(p)$, we have $q \in J^{+}(p)-I^{+}(p)$. Furthermore, any causal curve in $\bar{M}$ connecting $p$ to $q$ must lie entirely in $\dot{M}$ and, hence, must be a null geodesic in the spacetime $\left(\dot{M}, \tilde{g}_{a b}\right)$.

Proof: First, we claim that $A(p)$ is open in $\dot{M}$. Let $r \in A(p)$. Then there exists a causal curve $\lambda$ connecting $p$ to $r$ which - apart from its endpointslies in $M$. If $\lambda$ were not a null geodesic, we could deform it to a timelike curve in $M$ with the endpoints $p$ and $r$ fixed. However, if $\lambda$ is a null geodesic, then since $\Omega=0$ at its past and future endpoints on $\dot{M}, \lambda \cap M$ is a complete null geodesic in $\left(M, g_{a b}\right)$ (see eq.(D.6) of [2]). Since $\left(M, g_{a b}\right)$ satisfies the null energy condition and null generic condition, $\lambda$ must contain a pair of conjugate points. Therefore, in this case, $\lambda$ also can be deformed to be a timelike curve in $M$ with the endpoints $p$ and $r$ fixed. Thus $r \in I^{+}(p)$ and it follows that we can find a neighborhood of $r$ in $\dot{M}$ which is in $A(p)$, as we desired to show.

Now let $q \in \dot{A}(p)$. Clearly, $q \notin I^{+}(p)$, since otherwise an open neighborhood of $q$ would lie in $A(p)$. To show that $q \in J^{+}(p)$, let $\left\{q_{n}\right\}$ be a sequence of points in $A(p)$ which converges to $q$. For each $q_{n}$, let $\lambda_{n}$ be a causal curve connecting $p$ to $q_{n}$ which lies in $M$ apart from its endpoints. Since $q$ is a limit point of $\left\{\lambda_{n}\right\}$, we may apply the "limit curve" lemma (see, e.g., [9], [2]) to $\widetilde{M}-p$. It follows that in $\widetilde{M}$, there exists a past directed causal limit curve $\lambda$ through $q$ which either is past inextendible or has a past endpoint at $p$. Let $q^{\prime} \in I^{+}(q) \cap \dot{M}$. (Such a $q^{\prime}$ exists because $\dot{M}$ is timelike.) Then each $\lambda_{n}$ lies in $J^{+}(p) \cap J^{-}\left(q^{\prime}\right)$, which is compact, and hence is closed as a subset of $\widetilde{M}$. Consequently, since $\lambda$ is a limit curve of the sequence $\left\{\lambda_{n}\right\}$, we have $\lambda \subset J^{+}(p) \cap J^{-}\left(q^{\prime}\right)$. However, since $J^{+}(p) \cap J^{-}\left(q^{\prime}\right)$ is compact and $\bar{M}$ is strongly causal, $\lambda$ cannot be past inextendible (see, e.g., lemma 8.2 .1 of [Q] ]). Thus, $\lambda$ must have a past endpoint at $p$. Consequently, we have $q \in J^{+}(p)$, as we desired to show.

Finally, for $q \in \dot{A}(p)$, let $\tilde{\lambda}$ be any causal curve in $\bar{M}$ connecting $p$ with $q$. Suppose there exists a point $r \in \tilde{\lambda} \cap M$. Then there must exist an open segment of $\tilde{\lambda}$ contained in $M$ with endpoints on $\dot{M}$. By the same arguments as in the first paragraph of this proof, we can deform this segment of $\tilde{\lambda}$ to a timelike curve in $M$, keeping its endpoints fixed. We may then further deform $\tilde{\lambda}$ to a timelike curve in $M$ connecting $p$ with $q$. However, this contradicts the fact that $q \in \dot{A}(p)$. Thus, we have $\tilde{\lambda} \cap M=\emptyset$, so $\tilde{\lambda}$ must lie entirely in $\dot{M}$. Since $\left(\dot{M}, \tilde{g}_{a b}\right)$ is a spacetime in its own right, and since $q \in J^{+}(p)-I^{+}(p)$ 
[with $J^{+}(p)$ and $I^{+}(p)$ now defined with respect to the spacetime $\left(\dot{M}, \tilde{g}_{a b}\right)$ ] it follows that $\tilde{\lambda}$ must be a null geodesic with respect to $\tilde{g}_{a b}$.

It should be noted that anti-de Sitter spacetime satisfies all of the hypotheses of Theorem 2 except for the null generic condition. It also should be noted that anti-de Sitter spacetime fails to satisfy part of the conclusion of Theorem 2: $n$-dimensional anti-de Sitter spacetime is conformal to the region $0 \leq r<\frac{1}{2} \pi$ of the Einstein static universe

$$
d s^{2}=-d t^{2}+d r^{2}+\sin ^{2} r d \Omega^{2}
$$

Thus, $\dot{M}$ is comprised by the $(n-2)$-sphere $r=\frac{1}{2} \pi$. For $q \in \dot{A}(p)$ with $p$ and $q$ at antipodal points of this sphere, then in addition to null geodesics in $\dot{M}$ that connect $p$ with $q$, there also exist null geodesics in $M$ that connect $p$ with $q$ [15], in contradiction with the last sentence of Theorem 2. However, Theorem 2 implies that if we perturb anti-de Sitter spacetime in such a way that the geometry of $\dot{M}$ is not changed and in such a way that all of the hypotheses of Theorem 2 hold (including the null generic condition), then $p$ and $q$ no longer can be joined by a causal curve that enters $M$. Since the boundary geometry has not changed, we have a well defined, fixed "reference frame" with respect to which we can compare the causal properties of asymptotically anti-de Sitter spacetimes with that of anti-de Sitter spacetime itself. Thus, as already indicated in the Introduction, Theorem 2 may be interpreted as implying that the propagation of light through an asymptotically anti-de Sitter spacetime will always be "delayed" relative to propagation through anti-de Sitter spacetime itself.

\section{Acknowledgements}

This research was supported in part by NSF grant PHY 95-14726 to the University of Chicago.

\section{References}

[1] R.D. Reasenberg et al, Astrophys. J. Lett. 234, 219 (1979).

[2] R.M. Wald, General Relativity, University of Chicago Press (Chicago, 1984). 
[3] M. Visser, B. Bassett, and S. Liberati, Nucl. Phys. B88 (Proc. Supl.), 267 (2000), gr-qc/9810026; see also M. Visser, B. Bassett, and S. Liberati, in General Relativity and Relativistic Astrophysics, Proceedings of the Eighth Canadian Conference, ed. by C.P Burgess and R.C. Meyers, (AIP Press, Melville, New York, 1999), gr-qc/9908023.

[4] K.D.Olum, Phys.Rev.Lett. 81, 3567 (1998).

[5] R. Penrose, in Essays in General Relativity, ed. by F.J. Tipler, Academic Press (New York, 1980).

[6] R.P. Geroch and B.C. Xanthopoulos, J. Math. Phys. 19, 714 (1978).

[7] G.J. Galloway, math.DG/9909158.

[8] E. Woolgar, Class. Quant. Grav. 11, 1881 (1994).

[9] S.W. Hawking and G.F.R. Ellis, The Large Scale Structure of SpaceTime, Cambridge University Press (Cambridge, 1973).

[10] E.E. Flanagan, D. Marolf, and R.M. Wald, Phys. Rev. D (in press); hep-th/9908070.

[11] S. Kobayashi and K. Nomizu, Foundations of Differential Geometry, Volume I, Interscience Publishers (New York, 1963).

[12] N.J. Hicks, Notes on Differential Geometry, Van Nostrand (Princeton, 1965).

[13] R. Penrose, R. Sorkin, and E. Woolgar, gr-qc/9301015.

[14] A. Borde, Class. Quant. Grav. 4, 343 (1987).

[15] G. Horowitz and N. Itzhaki, JHEP 010, 9902 (1999). 